\title{
Estudio Fisicoquímico del Aceite y Análisis Proximal de la Torta de Semillas Oleaginosas nativas de Córdoba-Colombia
}

\author{
Jennifer J. Lafont, Amelia A. Espitia y Manuel S. Páez \\ Facultad de Ciencias Básicas, Dpto. de Química, Universidad de Córdoba - Colombia. \\ (e-mail: jenniferlafontmendoza@gmail.com)
}

Recibido Ago. 24, 2018; Aceptado Nov. 23, 2018; Versión final Ene. 24, 2019, Publicado Ago. 2019

\begin{abstract}
Resumen
El objetivo de este trabajo consistió en analizar las propiedades fisicoquímicas del aceite, perfil de ácidos grasos y el análisis proximal de la torta de tres semillas oleaginosas: Thevetia peruviana (calabonga), Gossypium hirsutum L (algodón) y Enterolobium ciclocarpum (orejero). Las semillas fueron recolectadas, extraídas sus nueces y trituradas para obtener el aceite respectivo por los métodos de prensado y solvente. Las propiedades fisicoquímicas se calcularon siguiendo la metodología de la Asociación Oficial de Químicos Analíticos (AOAC) y el perfil de ácidos grasos mediante cromatografía de gases/espectrometría de masas (GC/MS). La torta residual de la extracción por prensado fue empleada para el análisis proximal. Los resultados del aceite evidenciaron alto contenido de ácidos grasos insaturados (calabonga y algodón) clasificados como secantes, a diferencia del orejero con mayor proporción de saturados, no secantes. El análisis proximal de las tres tortas, demostró alto porcentaje de proteínas, teniendo el algodón mejor contenido nutricional. De acuerdo con los resultados se concluyó que las semillas en estudio pueden tener diversas aplicaciones en la industria.
\end{abstract}

Palabras clave: aceite; análisis fisicoquímicos; análisis proximal; torta; semillas oleaginosas

\section{Physicochemical Study of the Oil and Proximal Analysis of the Oilseed Cake Native to Córdoba-Colombia}

\begin{abstract}
The objective of this work was to analyze the physicochemical properties of the oil, fatty acid profile and the proximal analysis of the cakes of three oilseeds: Thevetia peruviana (calabonga), Gossypium hirsutum L (cotton) and Enterolobium cyclocarpum (orejero). The seeds were harvested, their nuts were extracted and crushed to obtain the respective oil by the methods of pressing and solvent. The physicochemical properties were calculated following the method of the Association of Official Analytical Chemists (AOAC) and the fatty acid profile by gas chromatography/mass spectrometry (GC/MS). The residual cake from the pressing extraction was used for the proximal analysis. The results of the oil showed high content of unsaturated fatty acids (calabonga and cotton) classified as drying, unlike orejero with a higher proportion of saturated, nondrying. The proximal analysis of the three cakes, showed a high percentage of proteins, the cotton having a better nutritional content. According to the results, it was concluded that the seeds under study may have diverse applications in the industry.
\end{abstract}

Keywords: oil; physicochemical analysis; proximal analysis; cake; oilseeds 


\section{INTRODUCCIÓN}

La Thevetia peruviana, comúnmente es denominada calabonga, es nativa de zonas tropicales de América, crece en forma silvestre en la Costa Caribe de Colombia, especialmente en el departamento de Córdoba, pertenece a la familia de las Apocináceas, es un arbusto que puede alcanzar hasta los 6 metros de altura, sus hojas son lanceoladas de color verde, de acuerdo con la variedad sus flores pueden ser amarillas (adelfa amarilla) o púrpuras (nerium oleander), ambas florecen y producen frutos de forma globular, con mesocarpio carnoso y diámetro de $4-5 \mathrm{~cm}$, suelen ser de color verde y se vuelven negros en la maduración; contiene entre una y cuatro semillas en su núcleo, se producen durante todo el año. La semilla contiene aceite y proteína, a pesar de ello, no son comestibles porque presentan glucósidos tóxicos al organismo, toxinas con acción cardíaca, el principio activo más poderoso es el thevetin, también se ha encontrado theveridosido, digitoxigenin, cerberin, peruvosido y theveside (Usman y otros, 2009) (Kumar y Sharma, 2011). En la literatura se han encontrado diversos estudios de la thevetia peruviana, algunos ensayan el aceite como suplemento alimenticio (Orozco Durán et al., 2016), otros para disminuir la metanogénesis entérica del ganado, (Hristov et al., 2013; Kumar et al., 2014). También consideran el aceite como una materia prima no comestible potencialmente importante para la producción de biodiesel de segunda generación (Atabani y otros, 2013; Kumar y Sharma, 2011).

El Gossypium hirsutum L, es una planta conocida comúnmente como algodón, pertenece a la familia malvaceae, se conocen 45 especies, las cuales pueden ser anuales, bianuales y perennes, herbáceas, arbustivas y arbóreas (Australian Government, 2008); puede alcanzar hasta $2.0 \mathrm{~m}$ de altura, la raíz principal es axonomorfa o pivotante, las secundarias crecen en dirección horizontal, el tallo principal es erguido, usualmente posee ramas vegetativas y reproductivas, las flores son dialipétalas, con 4 brácteas y estambres numerosos que envuelven al pistilo, la floración se presenta en el mes de agosto y la fructificación en los meses de noviembre-diciembre; el siguiente período de floración se lleva a cabo en febrero, es una planta autógama, aunque algunas flores abren antes de la fecundación, produciéndose semillas híbridas. El fruto es una cápsula que tiene entre tres y cinco carpelos, y de seis a diez semillas cada uno, (Fang y Percy, 2015). Su cultivo produce la fibra natural llamada algodón, utilizada por la industria textil, esta fibra se forma a partir de las células epidérmicas de las semillas, cuya longitud varía entre 20 y $45 \mathrm{~cm}$ (Australian Government, 2008); una vez retirada la fibra se pueden tomar las semillas, de las cuales se extrae un aceite de color rojo oscuro, debido a la presencia de un pigmento liposoluble denominado gosipol, que es un toxico específico para animales monogástricos, pero es destruido a nivel ruminal por los bovinos, haciéndose inactivo. Los estudios científicos de esta especie se han centrado en aumentar el rendimiento potencial de la fibra del algodón (Constable y Bange, 2015; Yang et al., 2014), al mejoramiento de la calidad de los cultivos, empleando prácticas de ingeniería genéticas (Maheshwari y Kovalchuk, 2014), la biotecnología, (Carlsson et al., 2014), los factores climáticos, tipos de suelos temperaturas y efectos adversos (Sawan, 2012; Sawan, 2018), así como la madurez fisiológica del cultivo y madurez de cosecha (Gwathmey, Bange y Brodrick, 2016). Otros investigadores recomiendan esta semilla para el uso de biocombustibles (Borugadda y Goud, 2012; Bergmann et al., 2013; Rozina, 2017; Kumar y Sharma, 2016).

El Enterolobium ciclocarpum, denominado popularmente orejero, crece en zonas tropicales y subtropicales secas, pertenece a la familia Fabaceae (mimosaceae), es un árbol de gran altura con copa globosa, sus hojas son verdes y bipinadas, las flores son actinomórficas, con corola verde clara, cáliz verde y tubular; sus semillas son de color marrón, grandes $(2.3$ por $1.5 \mathrm{~cm})$, ovoides y aplanadas, se encuentran dentro de vainas circulares indehiscentes, con forma arriñonada similar a una oreja de allí su nombre común, caen abundantemente del árbol las cuales son ingeridas por bovinos y equinos, (Martínez et al., 2012). Las investigaciones están orientadas a la floración y fructificación (Pineda et al., 2017), la evaluación del desarrollo del árbol en condiciones controladas (Hernández et al., 2014) y promover la germinación (Lozano et al., 2016). Las especies Thevetia peruviana, Gossypium hirsutum L y Enterolobium ciclocarpum, abundan en el departamento de Córdoba, y su utilidad es limitada; de acuerdo con la literatura consultada se encuentran muy pocos estudios sobre el aceite y la torta de estas materias primas, es por ello que el objetivo de este artículo estuvo encaminado a analizar las propiedades fisicoquímicas del aceite, su perfil de ácidos grasos y el contenido nutricional o análisis proximal de la torta de las tres semillas oleaginosas, con el fin de encontrar una posible aplicabilidad en la industria, para contribuir con el desarrollo de la región y del país. El mérito científico de este trabajo está orientado en dar conocer las propiedades de los aceites de algunas semillas poco conocidas, de abundante producción y uso limitado en las diversas industrias, así como el mayor aprovechamiento de la materia prima en el proceso de extracción del aceite por el método de prensado, obteniéndose el aceite virgen y como subproducto la torta residual que puede ser igualmente aprovechada en alimentos para animales, de acuerdo con su contenido nutricional. 


\section{METODOLOGÍA}

Las semillas fueron recolectadas en diferentes municipios del Departamento de Córdoba - Colombia, la calabonga en Planeta Rica, el algodón en Cereté, y el orejero en Lorica, luego fueron seleccionadas las de mejor estado físico y transportadas en neveras de icopor al laboratorio de Cinética y Biocombustibles de la Universidad de Córdoba. Las semillas de calabonga y orejero fueron descascaradas y secadas al sol para extraer la nuez; a las semillas de algodón inicialmente se les separó la mota o fibra, antes de ser asoleadas y extraídas sus nueces convirtiéndose en las materias primas de trabajo. Las muestras de Calabonga, algodón y orejero fueron maceradas, para extraer el aceite y realizar los análisis proximales respectivos.

Para extraer el aceite se emplearon dos métodos, el prensado mecánico donde la muestra macerada fue introducida en un dedal de tela fina, y se le aplicó una fuerza de compresión de $4000 \mathrm{Kg}$ (4 Ton), para extraer el aceite virgen. El segundo método fue el de extracción por solvente, para ello se empleó un equipo de soxhlet agregando el hexano en el balón, y la muestra macerada se transfirió a un cartucho poroso hecho de papel filtro que se ubicó en el dispositivo de extracción del soxhlet, de esta forma se realizó un reflujo sencillo durante 3 horas, pasado este tiempo se rota-evaporó, para recuperar el solvente y obtener el aceite. Posteriormente estos aceites fueron desgomados, en este paso se tomó la muestra y se sometió a calentamiento a una temperatura de $90^{\circ} \mathrm{C}$, agregándose el $10 \%$ de agua destilada con base al volumen de aceite, se dejó reposar por 30 minutos y posteriormente fue centrifugado a $4500 \mathrm{rpm}$ durante 10 minutos, obteniéndose 2 fases (aceite y gomas) las cuales fueron separadas por decantación reduciendo así las impurezas del mismo. A los aceites desgomados de las tres materias primas en estudio, se le determinaron algunas propiedades fisicoquímicas como: humedad, corrosión en lámina de cobre, número ácido, valores de yodo, peróxido y saponificación; para cada análisis fue utilizada una muestra de $5 \mathrm{~g}$ y se realizó por triplicado.

El contenido de humedad (Okonkwo y Okafor, 2016) fue determinado tomando una cápsula de porcelana previamente esterilizada, en la cual se pesó $5 \mathrm{~g}$ de la muestra $\left(\mathrm{P}_{\mathrm{m}}\right)$, esta fue introducida en una mufla marca $E \& Q$, durante 4 horas a la temperatura de $105^{\circ} \mathrm{C}$ luego fue retirada y pesada $\left(\mathrm{P}_{1}\right)$, posteriormente se colocó en un desecador y se pesó hasta un valor constante $\left(\mathrm{P}_{2}\right)$; el contenido de materia seca se calculó mediante la ecuación 1, donde Ms representa el porcentaje de materia seca, $\mathrm{P}_{1}$ es el peso inicial de la cápsula con la muestra húmeda, $\mathrm{P}_{2}$ es el peso final del crisol con la muestra seca, $\mathrm{Pm}$ es el peso de la muestra. El porcentaje de humedad $(\mathrm{H})$ se calculó de la diferencia de 100 con el contenido de matera seca (Ms).

$$
M s=\left[\frac{P_{2}-P_{1}}{P_{m}}\right] * 100 \%
$$

El número ácido, fue determinado pesando $5 \mathrm{~g}$ de aceite, a los cuales se le adicionó $50 \mathrm{~mL}$ de una solución etanol-éter dietílico en proporción 1:1 a temperatura ambiente y 3 gotas de fenolftaleína como indicador ácidobase, se tituló con hidróxido de potasio hasta el viraje de color; los ácidos grasos libres son expresados como ácido oleico (Okonkwo y Okafor, 2016). El valor de yodo se calculó pesando la muestra y disolviéndola en tetracloruro de carbono, luego se le agregó $25 \mathrm{~mL}$ del reactivo de Wijs y se dejó reposar la solución por 30 min en la oscuridad, pasado este tiempo se le agregó una solución de yoduro de potasio al 15\% y se tituló con tiosulfato de sodio $0.1 \mathrm{~N}$ utilizando almidón como indicador hasta que el color azul desapareció (Okonkwo y Okafor, 2016). El valor de peróxido, fue determinado agregando la muestra en una solución de ácido acéticocloroformo 3:2; luego fue adicionada una solución saturada de yoduro de potasio, posteriormente se tituló con tiosulfato de sodio $0.01 \mathrm{~N}$ utilizando almidón como indicador hasta que el color amarillo desapareció (AOCS Cd 8-53; 2003). El valor de saponificación fue calculado pesando $5 \mathrm{~g}$ de aceite, al cual se le agregó $25 \mathrm{~mL}$ de solución etanólica de hidróxido de potasio $0.5 \mathrm{~N}$ y sometido a reflujo durante $60 \mathrm{~min}$, posteriormente fue adicionado $1 \mathrm{~mL}$ de solución alcohólica de fenolftaleína al $1 \%$ y titulado con ácido clorhídrico $0.5 \mathrm{~N}$; también se realizó el blanco siguiendo el procedimiento anterior (AOCS Cd 3-25; 2003). Los ensayos de las muestras y del blanco se realizaron por triplicado. El análisis de corrosión en lámina de cobre (ASTM D130 -10; 2003) se realizó tomando la muestra de aceite a la que se le adicionó una lámina de cobre pulida, fueron calentados a 50ำ durante 180 minutos, después esta fue retirada y lavada con metanol, finalmente se comparó el color obtenido en la lámina calentada con el estándar de la norma para determinar el grado de corrosión.

Al aceite obtenido por prensado, también se le realizó la identificación del perfil de ácidos grasos, empleando un cromatógrafo de gases, Agilent $6890 \mathrm{~N}$ con detector selectivo de masas Agilent 5973N y Software - MSD Productivity Chemstation, con columna capilar Zebron ZB-5 (Phenomenex) de $30 \mathrm{~m}$ x $0.25 \mathrm{~mm}$ I.D, $0.25 \mu \mathrm{m}$ de espesor de película, detector FID, sistema de inyección Split. El procedimiento fue de inyección directa, previa dilución con isopropanol como solvente para volatilizar las muestras y facilitar su paso a través de la columna del equipo, por lo cual no fue necesario metilarlas. Para preparar las muestras (ASTM D-6584, 2003) se utilizó un vial para cromatografía marca AGILENT con volumen de $2 \mathrm{~mL}$, se le agregaron $50 \mu \mathrm{L}$ de aceite, 1 $\mu \mathrm{L}$ de estándar interno (n-tetradecano) y se adicionó isopropanol hasta aforar. De esta solución se tomó $1 \mu \mathrm{L}$ 
para ser inyectada en el cromatógrafo de gases acoplado a espectrometría de masas; con la ayuda de una jeringa Agilent con capacidad de 0-10 $\mu \mathrm{L}$; este ensayo se realizó por triplicado, es de anotar que antes de cada inyección se purgó la jeringa 10 veces con el solvente y 10 con la muestra. La identificación se realizó por comparación de los espectros de masas y los tiempos de retención de los compuestos identificados en el aceite, con las bases de datos NIST, WILEY Y ADAMS, que posee el equipo, con una correspondencia del $98 \%$. Las tortas residuales obtenidas en las extracciones por prensado de las tres materias primas, fueron secadas para evaluar su calidad nutricional, a través del análisis químico proximal, que involucra la determinación de la humedad, grasa cruda, cenizas (minerales totales), proteínas, fibra y carbohidratos; para ello se empleó la metodología de los Official Methods of Analysis de la AOAC (AOAC, 1995), siguiendo los ensayos analíticos de humedad $(930,15)$; proteína $(981,10)$; grasa $(960,39)$ y cenizas $(920,153)$; los carbohidratos fueron determinados mediante el método de la diferencia FAO, (2003). Es importante destacar que todos los ensayos tanto de las propiedades fisicoquímicas de los aceites como del análisis proximal de la torta en las tres materias primas fueron realizados por triplicado, para determinar el valor promedio y las desviaciones estándar para cada caso, las cuales son presentadas en las tablas de los resultados.

\section{RESULTADOS Y DISCUSIÓN}

Los resultados obtenidos de la extracción del aceite fueron mayores con el método del solvente, para las tres materias primas, calabonga $(76.48 \pm 0.20 \%)$, algodón $(77.79 \pm 1.46) \%$, y orejero $(72.50 \pm 0.34) \%$, con relación a la extracción por prensado que fueron de $(60.13 \pm 0.22) \%, \quad(62.37 \pm 0,19) \%$ y $(58.18 \pm 0.56) \%$ respectivamente, destacándose con mayor porcentaje el algodón. Las extracciones de aceite por el método de solvente extraen mayor cantidad de aceite de las semillas maceradas en los tres casos (calabonga, algodón y orejero), esto puede ser debido a que el solvente hexano es un hidrocarburo cuya estructura química no posee grupos funcionales, pero si una cadena carbonada similar a la de los compuestos que forman estos aceites, facilitando la salida de las moléculas oleaginosas presentes en la semilla durante el proceso de extracción; el solvente hexano penetra completamente en las células y tejidos vegetales de la semilla durante el reflujo sacando el máximo contenido de aceite en este proceso químico, dejando como subproducto una harina desgrasada; a diferencia de la extracción por prensado que presentó una eficiencia menor, lo cual puede ser debido a que en este proceso físico, la compresión rompe solamente las células y tejidos superficiales de la semilla, dejando retenido aceite en la torta residual. Sin embargo se puede destacar que ambos resultados de extracción de aceites tanto de solvente como de prensado, son buenos para realizar este proceso a escala industrial. En cuanto a los análisis fisicoquímicos reportados en la Tabla 1, se evidenciaron que las tres materias primas presentaron baja humedad, previniendo la descomposición del aceite mediante la proliferación de hongos y bacterias, además presentaron muy bajo poder de corrosivo (1A), siendo el aceite de algodón un poco mayor (1B), lo cual puede ser atribuido al incremento en el contenido de humedad, que facilita el aumento en la producción de ácidos grasos libres por hidrólisis de los triglicéridos, esto coincide con el mayor valor del numero ácido encontrado; sin embargo sigue siendo bajo el poder corrosivo, facilitando su almacenamiento.

El número ácido, es un indicador de la presencia de ácidos grasos libres, que aporta información sobre el grado de descomposición del aceite, (enranciamiento); este parámetro es usado para indicar el estado y comestibilidad de los mismos, el valor máximo permitido por la industria alimenticia para el aceite refinado es de $0.1 \mathrm{mg} \mathrm{KOH} / \mathrm{g}$ y sin refino es de $6.6 \mathrm{mg} \mathrm{KOH} / \mathrm{g}$, como ocurre en los aceites de estudio, presentando valores bajos, por lo tanto no se encontró enranciamiento y tienen buen estado de comestibilidad. El valor de yodo, es una medida del grado de insaturación del aceite, que puede ser clasificado como secantes (mayores a 140 meq $\mathrm{I}_{2} / \mathrm{g}$ ), semisecantes (110-139 meq $\mathrm{I}_{2} / \mathrm{g}$ ), y no secante (menores de $110 \mathrm{meq} \mathrm{I} / \mathrm{g}$ ), los aceites secantes son los que presentan alta proporción de ácidos grasos insaturados que cuando se polimerizan y secan con el oxígeno del aire, forman una película resistente y brillante; la velocidad de secado aumenta con el número de dobles enlaces. De acuerdo con los valores encontrados los aceites de calabonga y algodón se clasifican como secantes, dándole posibles usos para la industria de pinturas, barnices, y tintas; a diferencia del orejero que es un aceite no secante, lo cual le da características humectantes, útiles en la preparación de cremas para pieles secas, agrietadas, lociones, productos cosméticos y dermatológicos hidratantes.

El valor de peróxido, es un indicador del grado de oxidación o descomposición del aceite (rancidez) en el momento de la prueba; los peróxidos se forman cuando los ácidos grasos del aceite reaccionan con el oxígeno del aire, formando grados de enranciamiento, este estado se alcanza cuando los aceites son expuestos ya sea a la luz solar, a altas temperaturas o al aire por largo tiempo. Se considera que los aceites con valores superiores a 2 meq $\mathrm{O}_{2} / \mathrm{Kg}$ de muestra son más propensos a presentar rancidez (Godson et al., 2015), los resultados obtenidos en los aceites de la calabonga, algodón y orejero son menores que este valor, lo cual indica que son estables a la degradación. El valor de saponificación, es una medida usada para calcular el peso molecular de los ácidos grasos que contiene el aceite, por lo tanto a mayor longitud de cadena carbonada, se incrementará el valor de saponificación, lo cual se puede verificar con los resultados del perfil de ácidos grasos de la calabonga y el algodón que contienen ácidos grasos con 20 átomos de carbono, siendo 
cadenas carbonadas más largas que las presentes en el orejero. Los resultados de los análisis fisicoquímicos del aceite de algodón son comparables con otro estudio reportado por Okonkwo y Okafor, (2016), especialmente en los valores de saponificación $(193.31+0.31 \mathrm{mgKOH} / \mathrm{g})$ y valor ácido $(3.76+0.10 \mathrm{mg}$ $\mathrm{KOH} / \mathrm{g}$ ), los cuales son un poco menores que los obtenidos en este trabajo.

Tabla 1: Análisis fisicoquímicos de los aceites extraídos por prensado y solvente de las semillas en estudio

\begin{tabular}{|l|c|c|c|c|c|c|}
\hline \multirow{2}{*}{ Propiedad } & \multicolumn{2}{c|}{ Calabonga } & \multicolumn{2}{c|}{ Algodón } & \multicolumn{2}{c|}{ Orejero } \\
\cline { 2 - 7 } & Prensado & Solvente & Prensado & Solvente & Prensado & Solvente \\
\hline Humedad (\%) & $0.30 \pm 0.01$ & $0.60 \pm 0.01$ & $3.8 \pm 0.06$ & $4.3 \pm 0.08$ & $0.8 \pm 0.03$ & $1.0 \pm 0.01$ \\
\hline $\begin{array}{l}\text { Corrosión en lámina de } \\
\text { cobre }\end{array}$ & $1 \mathrm{~A}$ & $1 \mathrm{~A}$ & $1 \mathrm{~B}$ & $1 \mathrm{~B}$ & $1 \mathrm{~A}$ & $1 \mathrm{~A}$ \\
\hline Número ácido $(\mathrm{mg} \mathrm{KOH} / \mathrm{g})$ & $0.66 \pm 0.04$ & $1.17 \pm 0.04$ & $4.20 \pm 0.05$ & $5.80 \pm 0.08$ & $1.2 \pm 0.5$ & $1.5 \pm 0.04$ \\
\hline Valor de yodo $(\mathrm{meq} \mathrm{I} / \mathrm{g})$ & $168.20 \pm 0.40$ & $172.80 \pm 0.30$ & $186.50 \pm 0.09$ & $195.20 \pm 0.04$ & $103 \pm 0.03$ & $105 \pm 0.03$ \\
\hline $\begin{array}{l}\text { Valor de peróxido } \\
\text { (meq O2/Kg) }\end{array}$ & $1.20 \pm 0.01$ & $1.43 \pm 0.94$ & $1.66 \pm 0.15$ & $1.84 \pm 0.16$ & $0.50 \pm 0.12$ & $0.80 \pm 0.03$ \\
\hline $\begin{array}{l}\text { Valor de Saponificación } \\
\text { (mg KOH/g) }\end{array}$ & $198.00 \pm 0.03$ & $199.20 \pm 0.09$ & $180.90 \pm 0.30$ & $182.90 \pm 0.80$ & $92.0 \pm 0.15$ & $94.0 \pm 0.6$ \\
\hline
\end{tabular}

Los resultados del perfil de ácidos grasos de los tres aceites en estudio, se presentan en la Tabla 2, donde se observa que la calabonga y el algodón evidenciaron altos porcentajes de ácidos grasos insaturados, destacándose el oleico y linoléico respectivamente, además presentaron el ácido palmítico saturado; los mayores porcentajes de ácidos grasos insaturados favorecen la producción de biodiesel (Atabani et al., 2013). El resultado del aceite de orejero fue opuesto a los dos anteriores, el mayor porcentaje fue de ácidos grasos saturados, resaltando el palmítico y mirístico respectivamente, aunque también presentó el palmitoleico monoinsaturado en alta proporción; los altos porcentajes de ácidos grasos saturados en el aceite, lo convierte en un buen candidato para la producción de jabones y productos cosméticos. Estos resultados, están acordes con las propiedades fisicoquímicas, los cuales permiten sugerir posibles aplicabilidades industriales para estos aceites (Carlsson, et al, 2014), por ejemplo el de la calabonga y el algodón pueden ser potencialmente útiles en la industria de barnices, tintas, pinturas, biocombustibles (Bergmann et al, 2012) y el algodón además puede ser empleado como suplemento alimenticio para el ganado vacuno (Okonkwo y Okafor, 2016); el aceite de orejero por su parte sería más útil para la industria de alimentos y productos cosméticos humectantes y farmacológicos.

Tabla 2: Perfil de ácidos grasos de los aceites de calabonga, algodón y orejero

\begin{tabular}{|l|c|c|c|c|}
\hline Compuestos & $N^{\circ}$ C: doble enlace & Calabonga (\%) & Algodón (\%) & Orejero (\%) \\
\hline Ácido Palmitoléico & $\mathrm{C} 16: 1$ & $0.22 \pm 0.01$ & - & $28.25 \pm 0.09$ \\
\hline Ácido Palmítico & $\mathrm{C} 16: 0$ & $23.04 \pm 0.04$ & $23.43 \pm 0.06$ & $39.40 \pm 0.04$ \\
\hline Ácido Mirístico & $\mathrm{C} 14: 0$ & $0.21 \pm 0.03$ & - & $15.67 \pm 0.07$ \\
\hline Ácido Oleico & $\mathrm{C} 18: 1$ & $45.18 \pm 0.08$ & $15.36 \pm 0.03$ & - \\
\hline Ácido Linoléico & $\mathrm{C} 18: 2$ & $16.67 \pm 0.02$ & $50.48 \pm 0.09$ & $7.80 \pm 0.02$ \\
\hline Ácido Cáprico & $\mathrm{C} 10: 0$ & - & - & $3.78 \pm 0.03$ \\
\hline Ácido Láurico & $\mathrm{C} 12: 0$ & - & - & $3.12 \pm 0.05$ \\
\hline Ácido Esteárico & $\mathrm{C} 18: 0$ & $8.30 \pm 0.04$ & $2.21 \pm 0.04$ & $0.43 \pm 0.04$ \\
\hline Ácido Linolénico & $\mathrm{C} 18: 3$ & $0.20 \pm 0.02$ & $0.52 \pm 0.01$ & $1.13 \pm 0.08$ \\
\hline Ácido Araquídico & $\mathrm{C} 20: 0$ & $1.69 \pm 0.05$ & $0.10 \pm 0.07$ & - \\
\hline Total ácidos grasos saturados & 33.24 & 25.74 & 62.40 \\
\hline Total ácidos grasos insaturados & 62.27 & 66.36 & 37.18 \\
\hline
\end{tabular}

El perfil de ácidos grasos obtenido en el aceite de calabonga en este trabajo, es similar al reportado por Godson y Bassey, (2015), en el cual destaca el ácido oleico con mayor proporción (42.25)\%, seguido del 
palmítico (19.50)\%, linoléico (10.50)\%, esteárico (6.39)\%, mirístico (0.19)\% y linolénico (0.50)\%; principalmente, para un total de ácidos grasos saturados de $29.02 \%$ e insaturados de $53.68 \%$; también se encontraron valores cercanos en los trabajos realizados por Alabi, Lajide y Owolabi, (2013); Oseni, Obetta y Orukotan, (2012); Deka y Basumatary, (2011); a diferencia de Panchal et al., (2017) cuyos porcentajes se alejan significativamente, esto puede ser debido a las diferencias en la composición química del terreno donde fueron plantadas estas especies y las condiciones medioambientales, las cuales influyen el proceso bioquímico interno de las plantas para formar los ácidos grasos (Hernández et al., 2014). De igual forma los resultados de los porcentajes de ácidos grasos obtenidos en el aceite de orejero, son muy similares a los reportados por Hawas et al., (2012), donde se destaca un mayor contenido de ácidos grasos saturados con un total de $62.44 \%$, correspondientes al palmítico (38.89)\%, mirístico $(15.88) \%$, cáprico $(3.68) \%$, láurico (3.38)\%, y esteárico(0.61)\%; en menor proporción se encontraron los insaturados con un $37.51 \%$ formados por palmitoleico (28.99)\%, linoléico (7.57)\%, y linolénico $(0.95) \%$.

En la Tabla 3 se presentan los resultados de los análisis proximales de las tortas de las semillas de calabonga, algodón y orejero obtenidas de los procesos de extracción de aceites por prensado; como estas especies vegetales pertenecen a diversas familias, es de esperarse que también presenten resultados diferentes, como es el caso de la calabonga, cuyo porcentaje de extracción del aceite es cercano al del algodón, sin embargo la composición química de ambas semillas son diferentes, como se puede constatar en los resultados de los análisis proximales de sus tortas; sin embargo tienen tendencias similares de acuerdo con el método de extracción de aceite empleado. Se evidencian bajos contenidos de humedad, menores al $10 \%$, en las tres tortas, lo cual puede ser debido a las trazas de agua que no pudieron ser eliminadas en el proceso de prensado ya que no hubo calentamiento; este resultado quiere decir que poseen alto porcentaje de materia seca, esto favorece su conservación y almacenamiento, evitando la formación de hongos y micotoxinas que deterioran el producto. Las tres materias primas presentaron altos porcentajes de proteínas, excediendo lo requerido por la dieta del hombre y de los animales que es 19,8\%, según la FAO (Okonkwo y Okafor, 2016), siendo mayor para la calabonga, le sigue el orejero y por último el algodón, materias primas útiles para el crecimiento de animales, el método de extracción de aceites por prensado favorece que en la torta quede retenido mayor porcentaje de proteínas entre ellas las hidrofóbicas, capaces de atrapar moléculas de agua, evitando la desecación. El valor de cenizas representa la concentración de elementos minerales (macro y micro elementos) necesarios para la nutrición y crecimiento, están presentes en las tres semillas de estudio, destacándose la torta de calabonga con mayor proporción, el porcentaje de cenizas no es afectado por el método de extracción de aceite.

Tabla 3: Análisis proximal de las tortas de las semillas de calabonga, algodón y orejero

\begin{tabular}{|l|c|c|c|}
\hline Parámetro & Calabonga (\%) & Algodón (\%) & Orejero (\%) \\
\hline Humedad & $8.74 \pm 0.02$ & $8.50 \pm 0.12$ & $8.92 \pm 0.06$ \\
\hline Cenizas & $7.70 \pm 0.01$ & $6.90 \pm 0.06$ & $3.90 \pm 0.10$ \\
\hline Proteína cruda & $53.00 \pm 0.04$ & $24.50 \pm 0.05$ & $45.00 \pm 0.12$ \\
\hline Fibra & $2.59 \pm 0.08$ & $29.20 \pm 0.04$ & $0.84 \pm 0.10$ \\
\hline Grasa cruda & $4.93 \pm 0.19$ & $16.8 \pm 0.24$ & $4.80 \pm 0.10$ \\
\hline Carbohidratos & $23.04 \pm 0.47$ & $14.10 \pm 0.10$ & $36.54 \pm 0.09$ \\
\hline
\end{tabular}

La fibra es fundamental en la dieta porque mejora la digestión, facilita el tránsito intestinal, previene el estreñimiento y la distención abdominal, en humanos y animales; los valores de fibra revelan la presencia de carbohidratos de difícil digestión como la celulosa, hemicelulosa, pentosanos, lignina entre otros; los cuales quedan retenidos en mayor proporción en la torta residual obtenida de la extracción del aceite por prensado, a diferencia del método de solvente cuyos componentes son extraídos en los ciclos sucesivos de calentamiento con el soxhlet. En este sentido la torta de las semillas del algodón presentó mayor contenido de fibra (Shieunda, 2013). De las tres materias primas estudiadas, la torta de la semilla del algodón se destaca con alto contenido de fibra, proteína y grasa cruda en su composición, convirtiéndose en una excelente fuente alimenticia, debido a que rara vez se encuentran estos tres componentes altos en el mismo alimento. Shieunda, (2013), realizó una investigación en cuatro regiones geográficas de Kenia y determinó el análisis proximal de la semilla de calabonga, los resultados arrojaron valores de Humedad entre (1.71- 3.19)\%, cenizas (1.18 - 5.46)\%, proteína cruda $(28.23-30.0) \%$, fibra $(1.61-2.06) \%$, grasa cruda $(60.0-64.47) \%$ y carbohidratos $(0.73-4.30) \%$, se puede observar que la mayoría de los datos fueron mayores a los obtenidos por la torta residual en este trabajo, excepto el contenido de grasa total de la semilla que no se había extraído previamente. 
Esta diferencia en los valores del contenido nutricional, es variable dependiendo de los factores medioambientales, tipos de suelos, o estrés hídrico que esté sometida la planta (Hernández et al., 2014). En general se puede decir que el alto porcentaje de aceites extraídos por el método de solvente, de las semillas de Thevetia peruviana, Gossypium hirsutum $L$ y Enterolobium ciclocarpum, sus aceites vírgenes obtenidos por prensado y los excelentes resultados de los contenidos nutricionales (proteínas, fibra y grasa) de las tortas residuales, convierten a estas especies en materias primas potenciales para la optimización de los procesos a nivel industrial, con máximo aprovechamiento tanto del producto principal (aceites), como del producto secundario (torta residual), los cuales generan derivados que pueden ser comercializados, aumentando los ingresos y el desarrollo de la región.

\section{CONCLUSIONES}

De acuerdo con los resultados obtenidos se plantean las siguientes conclusiones: 1) El método de extracción de aceites a partir de las semillas de calabonga, algodón y orejero, con mayor rendimiento es el de solventes; 2) las propiedades fisicoquímicas del aceite y el perfil de ácidos grasos evidenciaron que los tres aceites son poco corrosivos, estables a la degradación y en su almacenamiento; 3) los aceites de calabonga y algodón son secantes y contienen mayor proporción de ácidos grasos insaturados, haciéndolos potencialmente útiles en la industria de pinturas, barnices, tintas y biocombustibles; mientras que el orejero es un aceite no secante, con mayor proporción de ácidos grasos saturados, lo cual facilita su posible uso en la industria cosmética y farmacológica; 4) del análisis proximal se concluye que las tres materias primas tienen alto contenido proteico, que puede ser aprovechable solo en las semillas de algodón y orejero; sin embargo la semilla de algodón tiene un contenido nutricional más completo, porque a diferencia de las otras presenta altos valores de fibra, proteína y grasa cruda convirtiéndola en un excelente suplemento alimenticio para bovinos.

\section{REFERENCIAS}

Alabi, K.A., L. Lajide y B.J. Owolabi, Analysis of Fatty Acid Composition of Thevetia peruviana and Hura crepitans Seed oils using GC-FID, Fountain Journal of Natural and Applied Sciences, 2(2), 32 - 37 (2013)

AOAC. Official Methods of Analysis. 16 $6^{\text {th }}$. Association of Official Analytical Chemists. Washington DC: AOAC International, 4-30 (1995)

AOCS Cd 3-25. Official Method Saponification Value, Sampling and Analysis of Commercial Fats and Oils. Copyright The American Oils Chemist's Society. Urbana - Illinois. USA (2003)

AOCS Cd 8-53. Official Method Peroxide Value Acetic Acid (Chloroform Method), Sampling and Analysis of Commercial Fats and Oils. Copyright The American Oils Chemist's Society. Urbana - Illinois. USA (2003)

ASTM D 6584. Standard Test Method for Determintion of Total Monoglyceride, Total Diglyceride, Total Triglyceride, and Free and Glycerin by Gas Cromatograpy (2003)

ASTM D130 - 10. Standard Test Method for Corrosiveness to Copper from Petroleum Products by Copper Strip Test. (2003)

Atabani, A.E., A.S. Silitonga y otros cinco autores, Non-edible vegetable oils: A critical evaluation of oil extraction, fatty acid compositions, biodiesel production, characteristics, engine performance and emissions production, doi: 10.1016/j.rser.2018.04.029, Renewable and Sustainable Energy Reviews, 18, 211-245 (2013)

Australian Government, The biology of Gossypium hirsutum L. and Gossypium barbadense L. (cotton), Office of the gene Technology regulator, Australia (2008)

Bergmann, J.C., D.D. Tupinamba y otros cuatro autores, Biodiesel production in Brazil and alternative biomass feedstocks, doi: 10.1016/j.rser.2018.04.029, Renewable and Sustainable Energy Reviews, 21411 - 420 (2013)

Borugadda, V.B. y V.V. Goud, Biodiesel production from renewable feedstocks: Status and opportunities, doi: 10.1016/j.rser.2018.04.029, Renewable and Sustainable Energy Reviews, 16(7), 4763-4784 (2012)

Carlsson, A.S., L.H. Zhu, Andersson y M. Hofvander, Platform crops amenable to genetic engineering - a requirement for successful production of bio - industrial oils through genetic engineering, doi: 10.1016 / j.nbt.2009.08.003, 3 Biocatalysis and Agricultural Biotechnology, (1), 58 - 64 (2014)

Constable, G.A. y M.P. Bange, The yield potential of cotton (Gossypium hirsutum L.), doi: 10.4172/2329-8863.1000373, Field Crops Research, 182, 98-106 (2015)

Deka, D.C. y S. Basumatary, High quality biodiesel from yellow oleander (Thevetia peruviana) seed oil, doi.org/10.1016/j.biombioe.2011.01.007, Biomass and Bioenergy, 35 (5), 1797-1803 (2011)

Fang, D.D. y R.G. Percy, Editores, Cotton, $2^{\text {th }}$ Agronomy monograph 57, doi: 10.2134 / agronmonogr57.frontmatter, American Society of Agronomy, Inc., Crop Science Society of America, Inc., and Soil Science Society of America, Inc., Madison (2015)

FAO, Food energy - methods of analysis and conversion factors, Food and Nutrition Paper 77, Report of a Technical Workshop, Food and Agriculture Organization of The United Nations, Rome (2003) 
Godson, A. y U. Bassey, Characterization of Oil and Biodiesel Produced from Thevetia peruviana (Yellow Oleander) Seeds, doi: 10.11648/j.jijse.20150404.14, International Journal of Sustainable and Green Energy, 4 (4), 150-158 (2015)

Gwathmey, C.O., M.P. Bange y R. Brodrick, Cotton crop maturity: A compendium of measures and predictors, doi: 10.4172/2329-8863.1000373, Field Crops Research, 191, 41-53 (2016)

Hawas, U.W., S.A. Eltomy y otros tres autores, Lipid content and antimicrobial activity of some Egyptian Fabaceae (Leguminosae) plants, doi: 10.5897/JMPR2018.6598, Journal of Medicinal Plants Research, 6(44), 5604-5608 (2012)

Hernández, M.L., B.L. Montalván y otros tres autores, Evaluación del desarrollo y crecimiento de Enterolobium cyclocarpum, Maclura tinctoria en condiciones controladas, Estelí -Nicaragua, doi: 10.5377/farem.v0i0.3109, Revista Científica de FAREM-Estelí. Medio ambiente, tecnología y desarrollo humano, 1(11), $69-80$ (2014)

Hristov, A.N., J. Oh y otros once autores, Special topics-mitigation of methane and nitrous oxide emissions form animal operations, review of enteric methane mitigation options, doi: 10.2527/jas.2016.1098, Journal of Animal Science, 91(11), 5045-5069 (2013)

Kumar, A. y S. Sharma, Potential non - edible oil resources as biodiesel feed - stock: an Indian perspective, doi: 10.1016/j.rser.2018.04.029, 15(4), Renewable and Sustainable Energy Reviews, 1791-1800 (2011)

Kumar, M. y M.P. Sharma, Selection of potential oils for biodiesel production, doi: 10.1016/j.rser.2018.04.029, Renewable and Sustainable Energy Reviews, 561129 - 1138 (2016)

Kumar, S., P.K. Choudhury y otros doce autores, New aspects and strategies for methane mitigation from ruminant, doi: 10.1007/s00253-017-8405-3, Applied Microbiology Biotechnology, 98(1), 31-44 (2014)

Lozano, E.C., M.A. Zapater y otros cuatro autores, Efecto de pretratamientos en semillas de Enterolobium contortisiliquum (Fabaceae) de la selva Pedemontana Argentina, Boletin de la Sociedad Argentina de Botánica, ISSN: 1851-2372, 51 (1), 79-87 (2016)

Maheshwari, P. e I. Kovalchuk, Genetic engineering of oil seed crops, doi: 10.1016 / j.nbt.2009.08.003, Biocatalysis and Agricultural Biotechnology, 3(1), 31 - 37(2014)

Martínez, M.M., R.E. Del Rio y otros cuatro autores, Enterolobium cyclocarpum (Jacq.) Griseb.:The biotechnological profile of a tropical tree, Boletín Latinoamericano y del Caribe de Plantas Medicinales y Aromáticas, ISSN: 0717-7917, 11 (5), $385-399(2012)$

Okonkwo, S.I. y E.C. Okafor, Determination of the Proximate Composition, Physicochemical Analysis and Characterization of Fatty Acid on the Seed and Oil of Gossypium Hirsutum, doi: 10.5539/ijc.v8n3p57, International Journal of Chemistry, 8 (3), 57-61 (2016)

Orozco, K.E., J. Herrera y otros cuatro autores, Reducción de la metanogénesis ruminal in vitro con aceites vegetales de Thevetia peruviana y Persea americana, doi: 10.19136/era.a5n13.1334, 3(9), Ecosistemas y recursos agropecuarios, 335$344(2016)$

Oseni, M.I., S.E. Obetta y F.V. Orukotan, Evaluation of fatty acids profile of ethyl esters of yellow oleander and groundnut oils as biodiesel feedstock, doi:10.5251/ajsir.2012.3.2.62.68, American Journal of Scientific and Industrial Research, 3 (2), 62-68 (2012)

Panchal, B.M., S.A. Deshmukh, D.S. Panchal y M.R. Sharma, Kinetics of the transesterification of non-edible Thevetia peruviana seed oil with dimethyl carbonate catalized by potassium methoxide, doi.org/10.1016/j.ejpe.2016.05.013, Egyptian journal of petroleum, 26, 363-370 (2017)

Pineda, E., J.I. Valdez., C.P. Pérez y R. Dávalos, Fenología, incremento en diámetro y periodicidad de Anillos de crecimiento de Enterolobium cyclocarpum (Jacq.) Griseb (Leguminosae) en Costa Grande, Guerrero, México, Polibotánica, ISSN: 1405-2768, 1(43), 1-21 (2017)

Rozina, S. Asif., M. Ahmad., M. Zafar y N. Ali, Prospects and potential of fatty acid methyl esters of some non-edible seed oils for use as biodiesel in Pakistan, doi: 10.1016/j.rser.2018.04.029, Renewable and Sustainable Energy Reviews, 74, 687-702 (2017)

Sawan, Z.M., Climatic variables: Evaporation, sunshine, relative humidity, soil and air temperature and its adverse effects on cotton production, doi: 10.1016/j.inpa.2016.10.005, Information Processing in Agriculture, 5(1), 134-148 (2018)

Sawan, Z.M., Cotton (Gossypium barbadense) production as affected by climatic factors and soil moisture status, doi: 10.1016 / j.chnaes.2016.12.009, Acta Ecologica Sinica, 32(1), 123-137 (2012)

Shieunda, O.R., Evaluation of Nuitritional Properties of Yellow Oleander (Thevetia Peruviana) Seeds in Kenya, Food Science and Quality Management, .22, 88-94 (2013)

Yang, Y., Y. Yang y otros tres autores, Prediction of cotton yield and water demand under climate change and future adaptation measures, Agricultural Water Management, ISSN: 0378-3774, 144, 42-53 (2014) 